\title{
Cavum septum pellucidum gigante y deterioro cognitivo en la esquizofrenia: análisis de un caso clínico.
}

\author{
Giant cavum septum pellucidum and cognitive impairment in schizophrenia: analysis of a \\ clinical case.
}

\begin{abstract}
Antonio González Moreno a, Daniel Gutiérrez Castillo ${ }^{\text {b }}$, Inmaculada Peñuelas Calvo ${ }^{\mathrm{c}}$.
${ }^{a}$ FEA de Psiquiatría. Coordinador de la Unidad de Salud Mental Las Lagunas de Fuengirola. Málaga, España. ${ }^{b}$ FEA de Psiquiatría. Unidad de Salud Mental Comunitaria del Valle del Guadalhorce. Málaga, España. ${ }^{c}$ MIR de Psiquiatría. Unidad de Salud Mental. Hospital Universitario Virgen de la Victoria. Málaga, España.
\end{abstract}

Correspondencia: Antonio González, Moreno (webantonio1@gmail.com)

Recibido: 09/08/2014; aceptado: 01/04/2015

RESUMEN: El cavum septum pellucidum (CSP) es
una alteración morfológica del septum pellucidum
que debe desaparecer entre los tres y los seis meses de
vida. Los CSP que persisten después de esa fecha y los
de tipo no comunicante se consideran una alteración
del neurodesarrollo y se han relacionado con la esqui-
zofrenia y otros trastornos psicóticos, especialmente
los de gran tamaño. La prevalencia es variable en fun-
ción del método de medida, de modo que encontramos
cifras que oscilan entre el $0,1 \%$ a $85 \%$ en la población
general y entre el 15 a $44,8 \%$ en la esquizofrenia. En
los pacientes con trastornos psicóticos y CSP grandes
se han observado una serie de características clínicas
tales como, mayor presencia de síntomas negativos,
alteraciones cognitivas del aprendizaje verbal y de la
comprensión de frases, entre otras. Presentamos el
caso clínico de una paciente de 19 años diagnosti-
cada de esquizofrenia hebefrénica en la que se halló
mediante tomografía computerizada (TC) un CSP
de tamaño gigante ( 2,6 cm) y en la que destaca la
importante presencia de síntomas negativos, el de-
terioro cognitivo, las alteraciones del aprendizaje
verbal y de la comprensión de frases e importantes
trastornos de conducta.
PALABRAS CLAVE: Cavum septum pellucidum,
esquizofrenia, deterioro cognitivo, neurodesarrollo.
ABSTRACT: Cavum septum pellucidum (CSP) is a morphological alteration of the septum pellucidum that should disappear at 3-6 months of age. CSPs that persist for longer, and non-communicating CSP, are classified as a neurodevelopmental disorders and have been associated with schizophrenia and other psychotic disorders, especially in cases with large CSPs. Prevalence rates vary according to the measurement method, and the figures range from $0,1 \%$ to $85 \%$ in the general population and from $15 \%$ to $44,8 \%$ in schizophrenia. A series of clinical features such as cognitive disorders, more negative symptoms and verbal learning and sentence comprehension disorders has been found in patients with psychotic disorders and large CSP. We present the clinical case of a 19-year old patient diagnosed with hebephrenic schizophrenia in which a CT scan revealed a giant CSP $(2,6 \mathrm{~cm})$ and who presented significant negative symptoms, cognitive deterioration, verbal learning and sentence comprehension disorders and important behavioural disorders.

KEY WORDS: Cavum septum pellucidum, schizophrenia, cognitive impairment, neurodevelopment.

\section{Introducción}

El cavum septum pellucidum (CSP) es una cavidad que se forma entre las dos hojas de dicho septum, situado en la parte media del encéfalo, entre la zona medial que forman los ventrículos laterales. El CSP se considera parte normal en el neurodesarrollo, estando presente en el $100 \%$ de los fetos, fusionándose entre los tres a seis 
meses de vida en más del $85 \%$ de los casos, persistiendo en aproximadamente un $10 \%$ de la población adulta (1).

En función del método de medida o resolución de la prueba de imagen (2), encontramos una prevalencia en la población de entre un 0,1 y $85 \%$. Según otros estudios (3), su prevalencia estimada en la esquizofrenia se sitúa entre 15 - 44,8\%.

Un reciente metaanálisis (4) afirma que no hay un incremento de la presencia de CSP de cualquier tamaño en los trastornos del espectro de la esquizofrenia. Sin embargo, sí es más común en estos pacientes el hallazgo de un CSP de gran tamaño, aunque se advierte de la posibilidad de que estudios con resultados negativos no hayan sido publicados.

Se ha relacionado el tamaño del CSP con alteraciones cognitivas. Así, en pacientes con CSP muy grandes (mayores de $6 \mathrm{~mm}$ ) (5) se ha hallado una presencia significativa de síntomas negativos, de alteraciones del aprendizaje verbal y de la comprensión de frases, así como de cociente intelectual bajo (5), mayores trastornos del pensamiento, mayor prevalencia de suicidios e importantes déficits cognitivos (5-6).

Presentamos un caso clínico que apoya las relaciones entre un CSP de gran tamaño $(2,6 \mathrm{~cm})$ y las alteraciones descritas en el artículo de Flashman y cols. (6), además de otras de las alteraciones características arriba descritas.

\section{Caso clínico}

Mujer de 19 años que realiza su tercer ingreso en Unidad de Hospitalización de Salud Mental por trastorno de conducta en el medio familiar.

Antecedentes familiares: Abuela con síndrome ansioso-depresivo. Madre: tentativa suicida mediante inhalación de gas cinco años antes y en tratamiento actual por síndrome ansioso-depresivo e hipotiroidismo.

Antecedentes personales: Oligoamnios, parto pretérmino, bajo peso al nacer (2070 g), icteria que requirió fototerapia, comunicación interventricular que cerró espontáneamente, clinodactilia de los dedos tercero, cuarto y quinto de ambos pies, heterocigótica para mutación 20210 del gen de la protrombina, cariotipo normal, dismorfia septal con insuficiencia velopalatina y rinitis hipertrófica con septoplastia y turbinoplastia. Deambuló a los nueve meses, controló esfínteres a los dos años y medio, fue autónoma para comer a los dos años y medio, para vestirse a los seis años y para asearse a los nueve. Levocardia-levoápex y situs solitus diagnosticado mediante ecografía.

Ha sido diagnosticada de trastorno psicótico agudo y transitorio (F23 CIE 10) y de esquizofrenia paranoide (F20 CIE 10), y desde los 16 años ha estado en seguimiento con Salud Mental Infanto-Juvenil. Estuvo en seguimiento en Neuropediatría por retraso madurativo y del lenguaje (empezó a hablar a los cuatro años, presentó 
NOTAS CLÍNICAS

dislexia que requirió tratamiento logopédico. A los siete años se midió la inteligencia por las matrices progresivas de Raven, que arrojaron cifras dentro del promedio, y a los diez años mediante WISC-R, obteniéndose los siguientes resultados: cociente intelectual verbal de 97, manipulativo de 72; la divergencia entre ambas cifras no permite establecer un cociente intelectual total, pero podríamos hablar de un índice general cognitivo correspondiente a una inteligencia media-baja.

Enfermedad actual: A su ingreso en la Unidad de Hospitalización, la paciente presentaba ideación delirante autorreferencial poco estructurada, alucinaciones auditivas comentadoras de su actividad, afectividad lábil y conducta pueril y regresiva con heteroagresividad en el medio familiar.

Las pruebas complementarias realizadas (bioquímica sanguínea con niveles de vitamina B12, ácido fólico y ceruloplasmina, análisis de orina, pruebas de función tiroidea, proteínas y electroencefalograma) fueron normales, salvo plaquetopenia fluctuante (que no llegó a normalizarse) y leucopenia. En la tomografía computarizada de cráneo se halla cavum septum pellucidum (ya detectado a los tres años de edad) de 2,6 cm de tamaño (Figura 1).

Figura 1:

TC craneal de la paciente donde puede observarse, delimitado por la circunferencia, el cavum septum pellucidum gigante. Autor: Antonio M. González Moreno

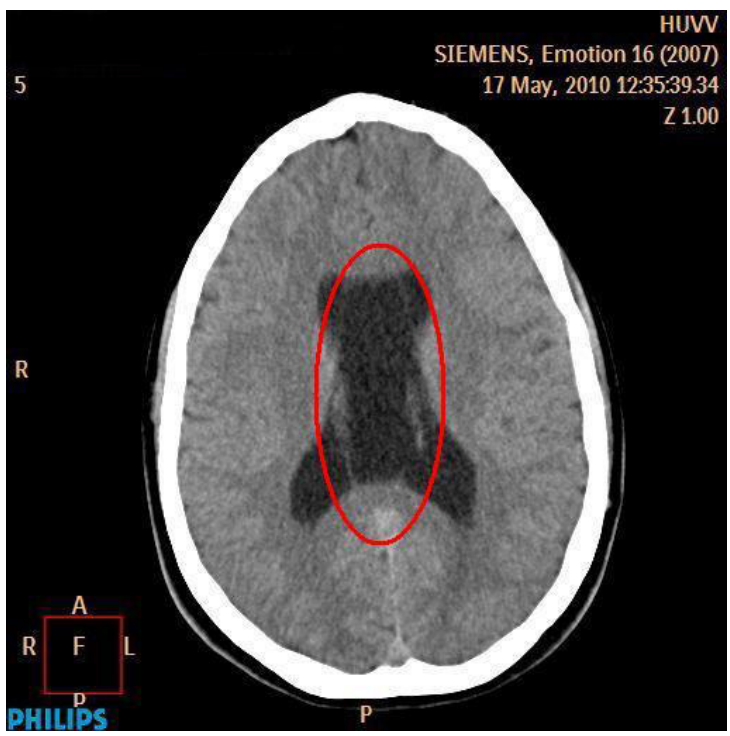


Se intentó realizar una evaluación de la inteligencia mediante WAIS-III, y aunque la paciente se mostró interesada, el test no pudo terminarse por la imposibilidad de obtener una valoración adecuada ya que daba respuestas tangenciales por asociaciones fonológicas y pararrespuestas. Al realizar las matrices de Raven, en lugar de seguir el orden lógico de la matriz agrupaba por colores o formas. Se achacaron las dificultades para entender y realizar la prueba a efectos secundarios cognitivos del topiramato, que tenía en su tratamiento, el cual se redujo progresivamente hasta la suspensión, y se intentó pasar el Test del Factor G de Cattell, obteniéndose resultados similares: la paciente no parecía comprender las instrucciones y daba respuestas tangenciales y no relacionadas con las preguntas. La impresión clínica era de un deterioro cognitivo moderado. Al mes de la suspensión total del topiramato y con posterioridad, ha seguido persistiendo la misma dificultad operativa ante pruebas neuropsicológicas. CIE10).

La paciente fue diagnosticada de esquizofrenia hebefrénica (F20.1 de la

\section{Discusión}

Consideramos que el caso que presentamos apoya la hipótesis desarrollada por Flashman y cols. en la que se relacionan la presencia de un CSP de gran tamaño (mayor o igual a $6 \mathrm{~mm}$ ) con síntomas negativos y alteración del aprendizaje verbal y de la comprensión de frases.

La paciente presentaba claros síntomas negativos (embotamiento afectivo, pobreza del habla y abulia) además de déficit de la comprensión verbal, no sólo por la impresión clínica, sino por la incapacidad de realizar las pruebas de inteligencia (incluso en las que no interviene el lenguaje como las matrices progresivas de Raven). Destacamos también en este caso el deterioro sufrido por la paciente a lo largo de los años tanto a nivel cognitivo como en la autonomía. Es un caso muy conocido por nosotros ya que durante cuatro años ha realizado seis ingresos. En el quinto presentó un cuadro de hipertiroidismo que precisó tratamiento corrector, y en el sexto se observa hipotiroidismo subclínico a pesar de la suspensión correcta del tratamiento antitiroideo. En la actualidad tiene 21 años y su deterioro cognitivo ha sido progresivo, así como las alteraciones conductuales, destacando un predominio de síntomas negativos que nos ha conducido a mantener el diagnóstico de esquizofrenia hebefrénica o desorganizada (F20.1 CIE 10), siendo inviables los intentos de realizar nuevas exploraciones neuropsicológicas e incluso una resonancia magnética nuclear cerebral dada la mala evolución y las dificultades que presentaba. 


\section{BIBLIOGRAFÍA}

(1) Crespo-Facorro B, Arango C, Bernardo M, Parellada E. Morfología cerebral en la esquizofrenia: estudios de neuroimagen estructural. En: Arango C, Crespo-Facorro B, Bernardo M, editores. Neuroimagen en psiquiatría. Barcelona: ArsMedica, 2003; p. 107-29.

(2) De Souza C. JA et al. Cavum septum pellucidum and adhesio interthalamica in schizophrenia and MRI study. Eur Psychiatry. 2006 Jul; 21(5): 291-9.

(3) Galarza M, Merlo AB, Ingratta A, Albanese AM. Cavum septum pellucidum and its increasd prevalence in schizophrenia: a neuroembryological classification. J Neuropsychiatry Cli Neurosci 2004; 16: 41-6.

(4) Trzesniak C, Oliveira IR, Kempton MJ, Galvão-de Almeida A, Chagas MH, Ferrari MC. Are cavum septum pellucidum abnormalities more common in schizophrenia spectrum disorders? A systematic review and meta-analysis. Schizophr Res. 2011 Jan;125(1):1-12.

(5) Nopoulos PC, Krie A, Andreasen NC. Enlarged cavum seti pellucidi in patients with schizophrenia: clinical and cognitive correlates. J Neuropsychiatry Clin Neurosci 2000; 12: 344-9.

(6) Flashman LA, Roth RM, Pixley HS, Cleavinger HB, McAllister TW, Vidaver R et al. Cavum septum pellucidum in schizophrenia: clinical and neuropsychological correlates. Psychiatry Res. 2007 Feb 28;154(2):147-55. 